\title{
INVERSE AUTOMATA AND MONOIDS AND THE UNDECIDABILITY OF THE CAYLEY SUBGRAPH PROBLEM FOR GROUPS
}

\author{
ANA OLIVEIRA* and PEDRO V. SILVA* \\ Centro de Matemática, Faculdade de Ciências, Universidade do Porto, 4099-002 Porto, Portugal \\ website:http://www.fc.up.pt/cmup
}

(Received 27 December, 1998)

\begin{abstract}
The structure of an inverse monoid can be determined by the complete set of Schützenberger graphs of a presentation. Necessary and sufficient conditions for a collection of inverse $\bar{X}$-graphs to be the complete set of Schützenberger graphs of some inverse monoid presentation are established and decidability results are obtained. Conditions for a single inverse $\bar{X}$-graph to be a Schützenberger graph for some presentation are also obtained, and both problems are restricted to the case of Clifford monoids and E-unitary inverse monoids. Decidability and undecidability results are obtained for the case of finite graphs. It is also proved that the problem of embedding a finite inverse $\bar{X}$-graph in the Cayley graph of a group is undecidable.
\end{abstract}

1991 Mathematics Subject Classification. 20M18, 20M35, 20F10.

1. Introduction. Each inverse monoid presentation $\operatorname{Inv}\langle X ; R\rangle$ induces a set of inverse graphs known as Schützenberger graphs. Each graph corresponds to a $\mathcal{D}$ class in the inverse monoid $M$ defined by the presentation. If $M$ is a group, we have a single Schützenberger graph which happens to be the well-known Cayley graph of the group. The straightforward generalization of Cayley graphs to inverse monoids wouldn't be adequate because the resulting graphs wouldn't be inverse in general. Therefore it is preferred to define the complete collection of Schützenberger graphs. In [8], Ruyle observed that the structure of $M$ is totally determined by the complete collection of Schützenberger graphs of the presentation $\operatorname{Inv}\langle X ; R\rangle$. Our first goal in this paper is to establish necessary and sufficient conditions for a collection of inverse $\bar{X}$-graphs to qualify as the complete set of Schützenberger graphs of some inverse monoid presentation. Some of the conditions we consider provide nice decidability algorithms for a finite collection of finite graphs. These results constitute the core of section 3 . In section 4 we consider particular classes of inverse monoids, namely Clifford monoids and E-unitary inverse monoids.

A related problem is to study under which conditions an inverse $\bar{X}$-graph qualifies as a Schützenberger graph of some inverse monoid presentation. This problem was solved for finite graphs by Cowan and Reilly [1], which actually produced an algorithm to decide this question. Ruyle studied the general case and produced necessary and sufficient conditions [8]. In section 5, we establish alternative conditions using the notation of section 3. Section 6 is devoted to this same problem when restricted to the case of Clifford monoids and E-unitary inverse monoids. Decidability for the case of a finite inverse $\bar{X}$-graph and Clifford monoids is also established. 
In section 7 we show that the latter problem is undecidable for the case of Eunitary inverse monoids. To prove this, we show that it is undecidable whether or not a finite inverse $\bar{X}$-graph embeds in the Cayley graph of a group, a problem of independent interest.

2. Preliminaries. We start by introducing some basic terminology and results from automata theory. For further information, the reader is referred to [2]. Let $X$ be a finite set, denoted usually in this context by alphabet. The free monoid on $X$ is denoted by $X^{*}$ and we define an $X$-language to be a subset of $X^{*}$. The empty word is denoted by 1 . Given $u, v \in X^{*}$, we say that $v$ is a factor (respectively prefix) of $u$ if $u=a v b$ (respectively $u=v b$ ) for some $a, b \in X^{*}$.

An $X$-graph is an ordered pair of the form $\Gamma=(V, E)$, where $V$ is a nonempty set and $E \subseteq V \times X \times V$. We call the elements of $V(\Gamma)=V$ vertices and the elements of $E(\Gamma)=E$ edges. An $X$-graph $\Gamma=(V, E)$ is said to be

- finite if $V$ is finite;

- deterministic if $(p, x, q),\left(p, x, q^{\prime}\right) \in E \Rightarrow q=q^{\prime}$.

A sequence of the form

$$
q_{0} \stackrel{x_{1}}{\longrightarrow} q_{1} \stackrel{x_{2}}{\longrightarrow} \ldots \stackrel{x_{n}}{\longrightarrow} q_{n}
$$

is said to be a path in $\Gamma$ if $\left(q_{j-1}, x_{j}, q_{j}\right) \in E$ for every $j \in\{1, \ldots, n\}$. The word $x_{1} \ldots x_{n}$ is the label of the path. If $n=0$, the path is trivial. If $q_{n}=q_{0}$, we call it a loop. A graph $\Gamma$ is called

- connected if, for all $p, q \in V$, there is a path in $\Gamma$ from $p$ to $q$.

If $\Gamma$ and $\Gamma^{\prime}$ are $X$-graphs, a graph homomorphism $\varphi: \Gamma \rightarrow \Gamma^{\prime}$ is a map $\varphi: V(\Gamma) \rightarrow V\left(\Gamma^{\prime}\right)$ such that $(p \varphi, x, q \varphi) \in E\left(\Gamma^{\prime}\right)$ whenever $(p, x, q) \in E(\Gamma)$. If $\varphi$ is an injective map, we have an embedding of graphs. If $\varphi$ is bijective and $\varphi^{-1}$ is also a homomorphism, then $\varphi$ is an isomorphism.

An $X$-automaton is a triple of the form $\mathcal{A}=(i, \Gamma, T)$, where $\Gamma$ is an $X$-graph, $i \in V(\Gamma)$ and $T \subseteq V(\Gamma)$. Two $X$-automata $\mathcal{A}=(i, \Gamma, T)$ and $\mathcal{A}^{\prime}=\left(i^{\prime}, \Gamma^{\prime}, T^{\prime}\right)$ are isomorphic if there exists a graph isomorphism $\varphi: \Gamma \rightarrow \Gamma^{\prime}$ satisfying $i \varphi=i^{\prime}$ and $T \varphi=T^{\prime}$. Let $\mathcal{A}=(i, \Gamma, T)$ be an $X$-automaton. We say that $\mathcal{A}$ is finite (respectively deterministic) if $\Gamma$ is finite (respectively deterministic). A path in $\mathcal{A}$ is a path in $\Gamma$. A path of the form (1) is said to be successful if $q_{0}=i$ and $q_{n} \in T$. The set of all labels of successful paths in $\mathcal{A}$ is the language recognized by $\mathcal{A}$ and is denoted by $L(\mathcal{A})$.

We say that an $X$-language $L$ is rational if $L=L(\mathcal{A})$ for some finite $X$-automaton $\mathcal{A}$. The terminology rational follows from a famous theorem by Kleene, that establishes an alternative characterization in terms of the so-called rational operators (see [2]). We denote the set of all rational $X$-languages by $\operatorname{Rat} X$.

We denote by $\bar{X}$ the set $X \cup X^{-1}$, where $X$ is a finite alphabet and $X^{-1}$ is a set of formal inverses of $X$. We define an involution on $\bar{X}^{*}$ by

$$
\left(x^{-1}\right)^{-1}=x, \quad\left(y_{1} \ldots y_{n}\right)^{-1}=y_{n}^{-1} \ldots y_{1}^{-1}
$$

for $x \in X$ and $y_{1}, \ldots, y_{n} \in \bar{X}$. The reduction map $\iota: \bar{X}^{*} \rightarrow \bar{X}^{*}$ assigns to every word $w \in \bar{X}^{*}$ the (unique) word obtained from $w$ by successively deleting from it all the factors of the form $y y^{-1}$, with $y \in \bar{X}$. 
An $\bar{X}$-graph is said to be inverse if

(i) $\Gamma$ is deterministic and connected;

(ii) $\forall p, q \in V(\Gamma) \forall y \in \bar{X}\left((p, y, q) \in E(\Gamma) \Rightarrow\left(q, y^{-1}, p\right) \in E(\Gamma)\right)$.

An inverse graph $\Gamma$ is called a tree if the empty word 1 is the unique reduced word labelling a loop in $\Gamma$. We say that an $\bar{X}$-automaton $\mathcal{A}=(i, \Gamma, T)$ is inverse (respectively a tree) if $\Gamma$ is inverse (respectively a tree) and $|T|=1$. It is common to replace $T$ by its unique element. We have the following. hold:

Proposition 2.1. [9] Given inverse $\bar{X}$-automata $\mathcal{A}$ and $\mathcal{B}$, the following conditions

(i) $\mathcal{A} \cong \mathcal{B}$ iff $L(\mathcal{A})=L(\mathcal{B})$.

(ii) There exists a homomorphism $\varphi: \mathcal{A} \rightarrow \mathcal{B}$ iff $L(\mathcal{A}) \subseteq L(\mathcal{B})$.

Next we introduce some basic tools from inverse semigroup theory. The reader is referred to [3] for general semigroup theory and to [7] for inverse monoids.

Let $M$ be a monoid. A relation $R$ on $M$ is assumed to be a subset $R \subseteq M \times M$. The congruence on $M$ generated by $R$ is denoted by $R^{\sharp}$. Given a congruence $\tau$ on $M$ and $a \in M$, we denote by $a \tau$ the congruence class of $a$.

The monoid $M$ is said to be inverse if

$$
\forall u \in M \quad \exists ! u^{-1} \in M: u u^{-1} u=u \text { and } u^{-1} u u^{-1}=u^{-1} .
$$

A commutative monoid whose elements are idempotents is said to be a semilattice. Such monoids constitute important examples of inverse monoids. Moreover, the subset of idempotents of an inverse monoid $M$ constitutes a semilattice. The semilattice of idempotents of $M$ is denoted by $E(M)$. We call $M$ a Clifford monoid if, for all $u \in M$ and $e \in E(M)$, we have that $e u=u e$.

If $M$ is an inverse monoid, there is a smallest congruence $\sigma$ on $M$ such that $M$ is a group; $\sigma$ is called the minimal group congruence on $M$. We say that $M$ is an $E$ unitary monoid if

$$
\forall u \in M \quad \forall e \in E(M) \quad(\text { ue } \in E(M) \Rightarrow u \in E(M)) .
$$

Equivalently, $M$ is E-unitary if $\sigma$ is idempotent-pure that is, if $u \sigma e$, with $u \in M$ and $e \in E(M)$, then $u \in E(M)$.

We also consider the natural partial order on an inverse monoid $M$. Given $a, b \in M$, we write $a \leq b$ if $a=e b$ for some $e \in E(M)$. Alternatively, $a \leq b$ if and only if $a a^{-1} b=a$.

The free inverse monoid on $X$ is defined as the quotient $\bar{X}^{*} / \rho$, where

$$
\rho=\left(\left\{\left(u u^{-1} u, u\right) \mid u \in \bar{X}^{*}\right\} \cup\left\{\left(u u^{-1} v v^{-1}, v v^{-1} u u^{-1}\right) \mid u, v \in \bar{X}^{*}\right\}\right)^{\sharp} .
$$

The congruence $\rho$ is known as the Vagner congruence on $\bar{X}^{*}$ and we denote the free inverse monoid on $X$ by $\operatorname{FIM}(X)$.

For every $w \in \bar{X}^{*}$, we define

$$
\operatorname{MT}(w)=\{u \iota \mid u \text { is a prefix of } w\} .
$$

The inverse tree automaton induced by $\operatorname{MT}(w)$ when we take 1 and $w \iota$ as initial and terminal vertices is called the Munn tree of $w$. Note that $\operatorname{MT}(w)$ defines a finite 
subtree of the standard Cayley graph of the free group on $X$. Munn trees play an essential role in the study of the free inverse monoid:

Proposition 2.2. [6] For all $u, v \in \bar{X}^{*}$, the following conditions are equivalent:

(i) $u \rho v$;

(ii) $\operatorname{MT}(u)=\operatorname{MT}(v)$ and $u \iota=v$;

(iii) the Munn trees of $u$ and $v$ are isomorphic.

A presentation of an inverse monoid is a formal expression of the form $\operatorname{Inv}\langle X ; R\rangle$, where $X$ is a nonempty set and $R$ is a relation on $\bar{X}^{*}$. If $X$ and $R$ are both finite, the presentation is said to be finite. The quotient $\bar{X}^{*} /(\rho \cup R)^{\sharp}$ is the inverse monoid defined by this presentation. It is a well-known fact that every inverse monoid can be defined by a presentation.

Let $\operatorname{Inv}\langle X ; R\rangle$ be an inverse monoid presentation and let $\tau=(\rho \cup R)^{\sharp}$. We can associate to every $w \in \bar{X}^{*}$ an inverse $\bar{X}$-graph $\mathcal{S} \Gamma(X, R, w)$ in a natural way. The set of vertices is the $\mathcal{R}$-class of $w \tau$ (see [3]) and the edges consist of all the triples of the form $(a, y, b)$ with $a, b \in \mathcal{R}_{w \tau}, y \in \bar{X}$ and $b=a(y \tau)$. The graph $\mathcal{S} \Gamma(X, R, w)$ is the Schützenberger graph of $w$ relative to the presentation $\operatorname{Inv}\langle X ; R\rangle$. When $M$ is a group, then $\mathcal{S}(X, R, w)$ is exactly the Cayley graph for the corresponding group presentation.

The $\bar{X}$-automaton $\left(\left(w w^{-1}\right) \tau, \mathcal{S} \Gamma(X, R, w), w \tau\right)$ is denoted by $\mathcal{S}(X, R, w)$. We say that $\mathcal{S}(X, R, w)$ is the Schützenberger automaton of $w$ relative to the presentation $\operatorname{Inv}\langle X ; R\rangle$. The relevance of the Schützenberger automata for the study of inverse monoid presentations is clear from the following results.

Proposition 2.3. [9]

$$
L(\mathcal{S}(X, R, w))=\left\{u \in \bar{X}^{*} \mid u \tau \geq w \tau\right\}=\left\{u \in \bar{X}^{*} \mid\left(w w^{-1} u\right) \tau=w \tau\right\} .
$$

Proposition 2.4. [9] Given $u, v \in \bar{X}^{*}$, the following conditions are equivalent:

(i) $u \tau=v \tau$;

(ii) $\mathcal{S}(X, R, u) \cong \mathcal{S}(X, R, v)$;

(iii) $L(\mathcal{S}(X, R, u))=L(\mathcal{S}(X, R, v))$.

3. The complete set of Schützenberger graphs. In this section, we give necessary and sufficient conditions for a set of inverse graphs to be the set of Schützenberger graphs of an inverse monoid presentation. Throughout this section, let $\left(\Gamma_{i}\right)_{i \in I}$ be a family of inverse $\bar{X}$-graphs. We define

$$
\Omega_{i}=\left\{L\left(p, \Gamma_{i}, q\right) \mid p, q \in V\left(\Gamma_{i}\right)\right\}
$$

and $\Omega=\cup_{i \in I} \Omega_{i}$. For every $u \in \bar{X}^{*}$, let

$$
\Omega(u)=\{L \in \Omega \mid u \in L\} .
$$

If $\Omega(u) \neq \emptyset$, let $L_{u}=\cap \Omega(u)$. Otherwise, let $L_{u}=\emptyset$. We consider the following condition:

(S) $\Omega=\left\{L_{u} \mid u \in \bar{X}^{*}\right\}$. 
Note that, by Proposition 2.4, condition $(S)$ could be expressed in terms of automata morphisms. In what follows, we will consider the two inclusions separately, namely,

$\left(S_{1}\right)$ For every language $L \in \Omega$, there is a word $u \in \bar{X}^{*}$ such that $L=L_{u}$.

$\left(S_{2}\right) \quad$ For every word $u \in \bar{X}^{*}, \Omega(u) \neq \emptyset$ and $L_{u} \in \Omega$.

The two following examples show that conditions $\left(S_{1}\right)$ and $\left(S_{2}\right)$ are independent. The set of graphs in Example 3.1 satisfies condition $\left(S_{1}\right)$ but doesn't satisfy condition $\left(S_{2}\right)$, while Example 3.2 provides the reverse situation.

EXAMPLE 3.1.
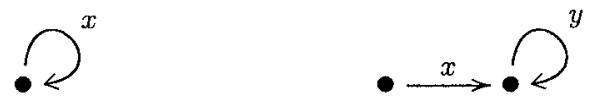

EXAMPLE 3.2 .
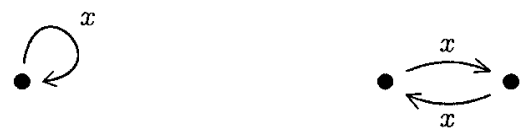

Lemma 3.3. If condition $\left(S_{2}\right)$ is satisfied, the relation $\tau$ defined on $\bar{X}^{*}$ by

$$
u \tau v \Leftrightarrow L_{u}=L_{v}
$$

is an inverse monoid congruence.

Proof. Suppose that condition $\left(S_{2}\right)$ is satisfied. Clearly, $\tau$ is an equivalence relation. Let $u, v, w \in \bar{X}^{*}$ be such that $u \tau v$, that is, $L_{u}=L_{v}$. By $\left(S_{2}\right), L_{u w} \in \Omega$; let $L_{u w}=L\left(p, \Gamma_{i}, q\right)$, with $p, q \in V\left(\Gamma_{i}\right)$. Hence $L_{u w} \neq \emptyset$ and so $u w \in L_{u w}$. Thus there is $r \in V\left(\Gamma_{i}\right)$ such that $u \in L\left(p, \Gamma_{i}, r\right)$ and $w \in L\left(r, \Gamma_{i}, q\right)$. It follows that $L\left(p, \Gamma_{i}, r\right) \in \Omega(u)$ and so $L_{u} \subseteq L\left(p, \Gamma_{i}, r\right)$. Since $L_{u}=L_{v}$, then $v \in L\left(p, \Gamma_{i}, r\right)$ so that $v w \in L\left(p, \Gamma_{i}, q\right)=L_{u w}$ and $L_{v w} \subseteq L_{u w}$. By symmetry, it follows that $L_{u w}=L_{v w}$, i.e., $(u w) \tau(v w)$. Similarly we get $(w u) \tau(w v)$ so that $\tau$ is a congruence on $\bar{X}^{*}$.

Note that, since the graphs $\Gamma_{i}$ are inverse then, for all $u, v \in \bar{X}^{*}$, we have that

$$
L_{u}=L_{u u^{-1} u} \text { and } L_{u u^{-1} v v^{-1}}=L_{v v^{-1} u u^{-1}}
$$

so that $\rho \subseteq \tau$ and $\tau$ is an inverse monoid congruence on $\bar{X}^{*}$.

Lemma 3.4. If condition $(S)$ is satisfied, the graphs $\left(\Gamma_{i}\right)_{i \in I}$ are, up to isomorphism, the set of Schützenberger graphs of the presentation $\operatorname{Inv}\langle X ; \tau\rangle$.

Proof. We begin by seeing that, for all $u \in \bar{X}^{*}, L_{u}=L(\mathcal{S}(X, \tau, u))$. By Proposition 2.4 , this is equivalent to

$$
L_{u}=\left\{v \in \bar{X}^{*} \mid u \tau=\left(u u^{-1} v\right) \tau\right\}
$$

and therefore to 


$$
L_{u}=\left\{v \in \bar{X}^{*} \mid L_{u}=L_{u u^{-1} v}\right\} .
$$

By condition $\left(S_{2}\right)$, we have $L_{u}=L\left(p, \Gamma_{i}, q\right)$, for some $p, q \in V\left(\Gamma_{i}\right)$. Suppose that $v \in L_{u}$; since $u \in L_{u}$ and $\Gamma_{i}$ is inverse, it follows that $u u^{-1} v \in L_{u}$ so that $L_{u u^{-1} v} \subseteq L_{u}$. Again, by condition $\left(S_{2}\right), L_{u u^{-1} v}=L\left(p^{\prime}, \Gamma_{j}, q^{\prime}\right)$ with $p^{\prime}, q^{\prime} \in V\left(\Gamma_{j}\right)$. Since $u u^{-1} v \in$ $L\left(p^{\prime}, \Gamma_{j}, q^{\prime}\right)$, then $u \in L\left(p^{\prime}, \Gamma_{j}, r\right)$, for some $r \in V\left(\Gamma_{j}\right)$. It follows that $L_{u} \subseteq L\left(p^{\prime}, \Gamma_{j}, r\right)$ which, since $v \in L_{u}$, gives that $v \in L\left(p^{\prime}, \Gamma_{j}, r\right)$. We then have that $u u^{-1} v \in L\left(p^{\prime}, \Gamma_{j}, r\right)$ and $u u^{-1} v \in L\left(p^{\prime}, \Gamma_{j}, q^{\prime}\right)$. Since $\Gamma_{j}$ is inverse, it follows that $r=q^{\prime}$ and so $u \in L_{u u^{-1} v}$ and $L_{u} \subseteq L_{u u^{-1} v}$. Hence $L_{u}=L_{u u^{-1} v}$.

Conversely, suppose that $v \in \bar{X}^{*}$ is such that $L_{u}=L_{u u^{-1} v}$. Since, by $\left(S_{2}\right)$, $u u^{-1} v \in L_{u u^{-1} v}$ then clearly $v \in L_{u u^{-1} v}$ i.e., $v \in L_{u}$. We have thus proved that $L_{u}=L(\mathcal{S}(X, \tau, u))$, for all $u \in \bar{X}^{*}$.

Let $\mathcal{S}$ be the set of Schützenberger graphs of the presentation $\operatorname{Inv}\langle X ; \tau\rangle$. We will see that, up to isomorphism, $\mathcal{S}=\left(\Gamma_{i}\right)_{i \in I}$. Let $u \in \bar{X}^{*}$ and $L_{u}=L\left(p, \Gamma_{i}, q\right)$, with $p, q \in V\left(\Gamma_{i}\right)$. From what we saw, $L_{u}=L(\mathcal{S}(X, \tau, u))$ which, since $\Gamma_{i}$ and $\mathcal{S} \Gamma(X, \tau, u)$ are inverse graphs, gives that $\mathcal{S}(X, \tau, u) \simeq\left(p, \Gamma_{i}, q\right)$ by Proposition 2.1 (i) and so $\mathcal{S} \Gamma(X, \tau, u) \simeq \Gamma_{i}$.

Conversely, given $\Gamma_{i}$ and $p, q \in V\left(\Gamma_{i}\right)$ we have, by $\left(S_{1}\right)$, that $L\left(p, \Gamma_{i}, q\right)=L_{u}$, for some $u \in \bar{X}^{*}$. Again, since $L\left(p, \Gamma_{i}, q\right)=L_{u}=L(\mathcal{S}(X, \tau, u))$ we have that $\left(p, \Gamma_{i}, q\right) \simeq \mathcal{S}(X, \tau, u)$ and $\Gamma_{i}$ is isomorphic to a graph in $\mathcal{S}$.

Theorem 3.5. A family $\left(\Gamma_{i}\right)_{i \in I}$ of inverse $\bar{X}$-graphs is the set of Schützenberger graphs of an inverse monoid presentation if and only if it satisfies condition (S).

Proof. To establish this result, it remains to see that condition $(S)$ is satisfied by the set of Schützenberger graphs of any inverse monoid presentation. Assume that $\left(\Gamma_{i}\right)_{i \in I}$ is the set of Schützenberger graphs of the presentation $\operatorname{Inv}\langle X ; R\rangle$. Then $\Omega=\left\{L(\mathcal{S}(X, R, u)) \mid u \in \bar{X}^{*}\right\}$. We show that $L_{u}=L(\mathcal{S}(X, R, u))$, for every $u \in \bar{X}^{*}$.

Let $u \in \bar{X}^{*}$. Since $u \in L(\mathcal{S}(X, R, u))$, we have $L(\mathcal{S}(X, R, u)) \in \Omega(u)$. Let $L \in \Omega(u)$, and let $v \in \bar{X}^{*}$ be such that $L=L(\mathcal{S}(X, R, v))$. Since $u \in L$, Proposition 2.4 yields $u \tau \geq v \tau$. Also, given $w \in L(\mathcal{S}(X, R, u))$, we have $w \tau \geq u \tau$, hence $w \tau \geq v \tau$ and so $w \in L$. Thus $L(\mathcal{S}(X, R, u)) \subseteq L$ and so $L(\mathcal{S}(X, R, u))=L_{u}$. Therefore $(S)$ holds.

We now give alternative conditions for $\left(S_{1}\right)$ and $\left(S_{2}\right)$. In view of these, it follows easily that, when we have a finite set of finite graphs, conditions $\left(S_{1}\right)$ and $\left(S_{2}\right)$ are decidable, and provide a simple algorithm to answer the problem.

Proposition 3.6. The following are equivalent:

(i) $\left(S_{1}\right)$;

(ii) for every $L \in \Omega$, we have that $L \nsubseteq \cup\left\{L^{\prime} \in \Omega \mid L \nsubseteq L^{\prime}\right\}$.

Proof. Suppose that $\left(S_{1}\right)$ holds, and let $L \in \Omega$. By $\left(S_{1}\right)$, there is $u \in \bar{X}^{*}$ such that $L=L_{u}$. Then $L=\cap \Omega(u)$ and $L \subseteq L^{\prime}$, for all $L^{\prime} \in \Omega(u)$. It follows that, if $L^{\prime} \in \Omega$ is such that $L \nsubseteq L^{\prime}$, then $L^{\prime} \notin \Omega(u)$, that is, $u \notin L^{\prime}$ and so $u \notin \cup\left\{L^{\prime} \in \Omega \mid L \nsubseteq L^{\prime}\right\}$. Since $u \in L$, then $L \nsubseteq \cup\left\{L^{\prime} \in \Omega \mid L \nsubseteq L^{\prime}\right\}$.

Conversely, suppose that condition (ii) is satisfied. Given $L \in \Omega$, there is $u \in \bar{X}^{*}$ such that $u \in L \backslash \cup\left\{L^{\prime} \in \Omega \mid L \nsubseteq L^{\prime}\right\}$. In particular, $\Omega(u) \neq \emptyset$. Let $L^{\prime \prime} \in \Omega(u)$. Since $u \in L^{\prime \prime}$ and $u \notin \cup\left\{L^{\prime} \in \Omega \mid L \nsubseteq L^{\prime}\right\}$, then $L^{\prime \prime} \nsubseteq \cup\left\{L^{\prime} \in \Omega \mid L \nsubseteq L^{\prime}\right\}$ so that $L \subseteq L^{\prime \prime}$. 
We then have that $L \in \Omega(u)$ and $L \subseteq L^{\prime \prime}$, for all $L^{\prime \prime} \in \Omega(u)$ which gives that $L=\cap \Omega(u)$ that is, $L=L_{u}$ and $\left(S_{1}\right)$ holds.

Proposition 3.7. Suppose that, for every word $u \in \bar{X}^{*}, \Omega(u) \neq \emptyset$. The following are equivalent:

(i) $\left(S_{2}\right)$;

(ii) for every $\Lambda \subseteq \Omega$ such that $\cap \Lambda \neq \emptyset$, there is $\Lambda^{\prime} \subseteq \Omega$ such that $\cup \Lambda^{\prime}=\cap \Lambda$.

Proof. Suppose that $\left(S_{2}\right)$ is satisfied, and let $\Lambda \subseteq \Omega$ be such that $\cap \Lambda \neq \emptyset$. For every $u \in \cap \Lambda$, we have that $L_{u} \subseteq \cap \Lambda$ and it follows that, if $\Lambda^{\prime}=\left\{L_{u} \mid u \in \cap \Lambda\right\}$, then $\cup \Lambda^{\prime}=\cap \Lambda$.

Conversely, assume that condition (ii) is satisfied, and let $u \in \bar{X}^{*}$. We have that $\Omega(u) \neq \emptyset$ and, since $u \in \cap \Omega(u)$, then $\cap \Omega(u) \neq \emptyset$. By condition (ii), there exists $\Lambda^{\prime} \subseteq \Omega$ such that $\cup \Lambda^{\prime}=\cap \Omega(u)$. It follows that $u \in \cup \Lambda^{\prime}$ so that $u \in L$, for some $L \in \Lambda^{\prime}$. Thus $L \in \Omega(u)$ and hence $\cap \Omega(u) \subseteq L$. On the other hand $L \in \Lambda^{\prime}$ gives that $L \subseteq \cup \Lambda^{\prime}=\cap \Omega(u)$. Hence $L=\cap \Omega(u)=L_{u}$ and $L_{u} \in \Omega$.

COROllary 3.8. Suppose that, for every word $u \in \bar{X}^{*}, \Omega(u) \neq \emptyset$ and that $\Omega$ is finite. The following are equivalent:

(i) $\left(S_{2}\right)$;

(ii) for every $L, L^{\prime} \in \Omega$ such that $L \cap L^{\prime} \neq \emptyset$, there are $L_{1}, \ldots, L_{k} \in \Omega$ such that $\cup_{i} L_{i}=L \cap L^{\prime}$.

Proof. Note that, when $\Omega$ is finite, Proposition 3.7 gives that condition (i) is equivalent to the following condition:

$$
\forall L^{(1)}, \ldots, L^{(n)} \in \Omega\left(\cap_{j} L^{(j)} \neq \emptyset \Rightarrow \exists L_{1}, \ldots, L_{k} \in \Omega: \cup_{i} L_{i}=\cap_{j} L^{(j)}\right) .
$$

It is clear that condition (2) implies condition (ii). To prove the reverse we use induction on $n$. For $n=1,2$ (ii) implies (2) trivially. We suppose now that (ii) implies (2), for all values smaller than $n$. Let $L^{(1)}, \ldots, L^{(n)} \in \Omega$ be such that $\cap_{j=1}^{n} L^{(j)} \neq \emptyset$. Then $\cap_{j=1}^{n-1} L^{(j)} \neq \varnothing$ and so, by the induction hypothesis, $\cap_{j=1}^{n-1} L^{(j)}=L_{1} \cup \ldots \cup L_{k}$, for some $L_{1}, \ldots, L_{k} \in \Omega$. Then

$$
\cap_{j=1}^{n} L^{(j)}=\left(L_{1} \cup \ldots \cup L_{k}\right) \cap L^{(n)}=\left(L_{1} \cap L^{(n)}\right) \cup \ldots \cup\left(L_{k} \cap L^{(n)}\right) .
$$

Also, for each $i=1, \ldots k$,

$$
L_{i} \cap L^{(n)}=L_{1}^{(i)} \cup \ldots \cup L_{k_{i}}^{(i)}
$$

for some $L_{1}^{(i)}, \ldots, L_{k_{i}}^{(i)} \in \Omega$. Since $\cap_{j=1}^{n} L^{(j)} \neq \emptyset$, then $L_{i} \cap L^{(n)} \neq \emptyset$, for some $i=1 \ldots k$, and the result follows by induction.

Given a finite set $\left\{\Gamma_{1}, \ldots, \Gamma_{n}\right\}$ of finite inverse $\bar{X}$-graphs, it is thus possible to decide whether or not $\left\{\Gamma_{1}, \ldots, \Gamma_{n}\right\}$ is the set of Schützenberger graphs of an inverse monoid, as we next describe. Since the graphs $\Gamma_{i}$ are finite, we have $\Omega \subseteq \operatorname{Rat} \bar{X}$. We make use of the following well-known facts about rational languages [2].

- If $L, L^{\prime} \in \operatorname{Rat} \bar{X}$, then $L \cup L^{\prime}$ and $L \cap L^{\prime}$ are effectively constructible rational $\bar{X}$-languages.

- If $L, L^{\prime} \in \operatorname{Rat} \bar{X}$, it is decidable whether or not $L \subseteq L^{\prime}$. 
To have $\Omega(u) \neq \emptyset$ for every $u \in \bar{X}^{*}$ is equivalent to the inclusion $\bar{X}^{*} \subseteq \cup \Omega$ and so we can decide whether or not this condition holds, a necessary condition for $\left(S_{2}\right)$ to hold. If this condition holds then Corollary 3.8 yields that $\left(S_{2}\right)$ is decidable. It follows from Proposition 3.6 that $\left(S_{1}\right)$ is also decidable.

4. E-unitary and Clifford monoids. In this section, we provide necessary and sufficient conditions for a set of inverse graphs to be the set of Schützenberger graphs of an E-unitary monoid and of a Clifford monoid. Given an $\bar{X}$-language $L$, we denote by $\operatorname{Pref}(L)$ the set of prefixes of the words of $L$.

Let $\left(\Gamma_{i}\right)_{i \in I}$ be a family of inverse $\bar{X}$-graphs satisfying condition $(S)$. Let $\tau$ be the congruence on $\bar{X}^{*}$ defined by $u \tau v \Leftrightarrow L_{u}=L_{v}$, and let $M$ be the inverse monoid determined by the presentation $\operatorname{Inv}\langle X ; \tau\rangle$. Recall that, by Lemma 3.4, we have that $\left(\Gamma_{i}\right)_{i \in I}$ is the set of Schützenberger graphs of the presentation $\operatorname{Inv}\langle X ; \tau\rangle$.

From [5], we have that $M$ is E-unitary if and only if the natural morphism associated with $\sigma$ induces a graph embedding of each $\Gamma_{i}$ into the Cayley graph of the maximal group homomorphic image of $M$ (relative to the induced set of generators). Next we give several other equivalent conditions.

THEOREM 4.1. If $(S)$ holds, the following conditions are equivalent:

(i) the monoid $M$ is E-unitary;

(ii) for all $L, L^{\prime} \in \Omega, \quad\left(\left(1 \in L \wedge L^{\prime} \subseteq L\right) \Rightarrow 1 \in L^{\prime}\right)$;

(iii) for all $i, j \in I, \quad\left(L\left(p, \Gamma_{i}, p\right) \cap L\left(q, \Gamma_{j}, r\right) \neq \emptyset \Rightarrow q=r\right)$;

(iv) all graphs $\Gamma_{i}$ embed in the Cayley graph of some group.

Proof. We begin by showing that (i) $\Leftrightarrow$ (ii). It is straightforward to verify that, for all $u \in \bar{X}^{*}, u \tau$ is idempotent if and only if $1 \in L(S(X, \tau, u))$. We have that $M$ is $\mathrm{E}$ unitary if and only if, for all $u, e \in \bar{X}^{*}, u \tau \geq e \tau$ and $e \tau$ idempotent imply that $u \tau$ is idempotent. Let $u, v \in \bar{X}^{*}$. By Proposition 2.3 and condition $(S)$,

$$
\begin{aligned}
u \tau \geq v \tau & \Leftrightarrow u \in L(\mathcal{S}(X, \tau, v)) \\
& \Leftrightarrow u \in L_{v} \\
& \Leftrightarrow L_{u} \subseteq L_{v} .
\end{aligned}
$$

It follows that $M$ is E-unitary if and only if, for all $u, v \in \bar{X}^{*}, L_{u} \subseteq L_{v}$ and $1 \in L_{v}$ imply that $1 \in L_{u}$. Now condition $(S)$ yields the equivalence (i) $\Leftrightarrow$ (ii).

By [5], we have that (i) $\Rightarrow$ (iv). Suppose that (iv) holds so that for all $i$, there is an embedding $\varphi_{i}: \Gamma_{i} \rightarrow \Gamma^{\prime}$, where $\Gamma^{\prime}$ is the Cayley graph of some group. Let $u \in L\left(p, \Gamma_{i}, p\right) \cap L\left(q, \Gamma_{j}, r\right)$. Since $u \in L\left(p, \Gamma_{i}, p\right)$, then $u$ labels a loop in $\Gamma_{i}$ and so $u$ labels a loop at every vertex in $\Gamma^{\prime}$. Then $q \varphi_{j}=r \varphi_{j}$ which, since $\varphi_{j}$ is injective, gives that $q=r$ and so (iii) holds. Hence (iv) $\Rightarrow$ (iii).

Now, given (iii), let $L, L^{\prime} \in \Omega$ be such that $1 \in L$ and $L^{\prime} \subseteq L$. Since $1 \in L$ then $L=L\left(p, \Gamma_{i}, p\right)$ for some $i$ and some $p \in V\left(\Gamma_{i}\right)$. Let $L^{\prime}=L\left(q, \Gamma_{j}, r\right)$ for some $j$ and $q, r \in V\left(\Gamma_{j}\right)$. As $L^{\prime} \subseteq L$, we have that $L\left(p, \Gamma_{i}, p\right) \cap L\left(q, \Gamma_{j}, r\right) \neq \emptyset$ which, by hypothesis, gives that $q=r$. Hence $1 \in L\left(q, \Gamma_{j}, r\right)=L^{\prime}$, and the result follows.

THEOREM 4.2. If $(S)$ holds, the following conditions are equivalent:

(i) $M$ is a Clifford monoid;

(ii) for all $i \in I$ and all $L, L^{\prime} \in \Omega_{i}, \operatorname{Pref}(L)=\operatorname{Pref}\left(L^{\prime}\right)$; 
(iii) for all $i \in I$ and all $p, q \in V\left(\Gamma_{i}\right), L\left(p, \Gamma_{i}, p\right)=L\left(q, \Gamma_{i}, q\right)$.

Proof. The inverse monoid $M$ is a Clifford monoid if and only if $\mathcal{D} \subseteq \mathcal{R}$. We have that, for all $u, v \in \bar{X}^{*}$,

$$
u \tau \mathcal{R} v \tau \Leftrightarrow\left(u u^{-1}\right) \tau\left(v v^{-1}\right) \Leftrightarrow L_{u u^{-1}}=L_{v v^{-1}}
$$

and, clearly,

$$
L_{u u^{-1}}=L_{v v^{-1}} \Rightarrow \operatorname{Pref}\left(L_{u}\right)=\operatorname{Pref}\left(L_{u u^{-1}}\right)=\operatorname{Pref}\left(L_{v v^{-1}}\right)=\operatorname{Pref}\left(L_{v}\right) .
$$

So if $M$ is a Clifford monoid, $u \tau \mathcal{D} v \tau$ implies that $\operatorname{Pref}\left(L_{u}\right)=\operatorname{Pref}\left(L_{v}\right)$, for all $u, v \in \bar{X}^{*}$. On the other hand, note that, if $i \in I$ and $L, L^{\prime} \in \Omega_{i}$, then $L=L_{u}$ and $L^{\prime}=L_{v}$, with $u, v \in \bar{X}^{*}$ such that $u \tau \mathcal{D} v \tau$. It thus follows that, if $M$ is a Clifford monoid, then, for all $i \in I$ and $L, L^{\prime} \in \Omega_{i}$, we have that $\operatorname{Pref}(L)=\operatorname{Pref}\left(L^{\prime}\right)$ that is, (i) $\Rightarrow$ (ii).

Let $i \in I$. Assume that (ii) holds, and let $p, q \in V\left(\Gamma_{i}\right)$. We have that $L\left(p, \Gamma_{i}, p\right)=L_{e}$, for some $e \in \bar{X}^{*}$. By assumption, $\operatorname{Pref}\left(L\left(p, \Gamma_{i}, p\right)\right)=\operatorname{Pref}\left(L\left(q, \Gamma_{i}, q\right)\right)$ which, as $e \in \operatorname{Pref}\left(L\left(p, \Gamma_{i}, p\right)\right)$, gives that $e \in \operatorname{Pref}\left(L\left(q, \Gamma_{i}, q\right)\right)$. Hence $e \in L\left(q, \Gamma_{i}, r\right)$, for some $r \in V\left(\Gamma_{i}\right)$ and thus $L\left(p, \Gamma_{i}, p\right)=L_{e} \subseteq L\left(q, \Gamma_{i}, r\right)$. Since $1 \in L\left(p, \Gamma_{i}, p\right)$, it follows that $r=q$ and so $e \in L\left(q, \Gamma_{i}, q\right)$, yielding that $L_{e}=L\left(p, \Gamma_{i}, p\right) \subseteq L\left(q, \Gamma_{i}, q\right)$. Now (iii) follows by symmetry.

Now, suppose we have (iii). Let $u, e \in \bar{X}^{*}$ be such that $e \tau$ is idempotent. Let $i \in I$. If $e$ labels a path in $\Gamma_{i}$ then $e \in L\left(p, \Gamma_{i}, q\right)$, for some $p, q \in V\left(\Gamma_{i}\right)$. Since $1 \in L_{e}$, we must have $q=p$. By (iii), this implies that $e \in L\left(p, \Gamma_{i}, p\right)$, for all $p \in V\left(\Gamma_{i}\right)$. We then have that $u e \in L\left(p, \Gamma_{i}, q\right)$ if and only if $e u \in L\left(p, \Gamma_{i}, q\right)$, for all $p, q \in V\left(\Gamma_{i}\right)$. It follows that $\Omega(u e)=\Omega(e u)$ so that $L_{u e}=L_{e u}$ and $(u e) \tau=(e u) \tau$. Hence $M$ is a Clifford monoid and the proof is complete.

5. Characterizing Schützenberger graphs. In [8], Ruyle characterized those inverse $\bar{X}$-graphs which arise as Schützenberger graphs for some inverse monoid presentation, using endomorphisms of graphs and orbits. In this section, we provide alternative characterizations using the notation introduced in section 3 .

If $\Gamma$ is an inverse $\bar{X}$-graph, let $\Omega$ denote the set

$$
\Omega=\{L(p, \Gamma, q) \mid p, q \in V(\Gamma)\} .
$$

As before, for every $u \in \bar{X}^{*}$, let

$$
\Omega(u)=\{L \in \Omega \mid u \in \Omega\} .
$$

If $\Omega(u) \neq \emptyset$, let $L_{u}=\cap \Omega(u)$; otherwise, let $L_{u}=\emptyset$. We consider the following condition:

$\left(S^{\prime}\right) \quad$ There is $u \in \bar{X}^{*}$ such that $L_{u} \in \Omega$.

THeOREM 5.1. Let $\Gamma$ be an inverse $\bar{X}$-graph. The following conditions are equivalent:

(i) $\Gamma$ is a Schützenberger graph of some inverse monoid presentation;

(ii) $\left(S^{\prime}\right)$;

(iii) there is $L \in \Omega$ such that $L \nsubseteq \cup\left\{L^{\prime} \in \Omega \mid L \nsubseteq L^{\prime}\right\}$. 
Proof. We show first that (i) $\Leftrightarrow$ (ii). The implication (i) $\Rightarrow$ (ii) follows easily from Theorem 3.5. Conversely, suppose we have (ii) and let $p, q \in V(\Gamma)$ be such that $L_{u}=L(p, \Gamma, q)$. Let $\sim_{u}$ be the syntactic congruence of $L_{u}$ on $\bar{X}^{*}$ that is, $w \sim_{u} w^{\prime}$ if and only if, for all $a, b \in \bar{X}^{*}, a w b \in L_{u} \Leftrightarrow a w^{\prime} b \in L_{u}$. We are going to prove that $(p, \Gamma, q) \simeq \mathcal{S}\left(X, \sim_{u}, u\right)$, by showing that

$$
L_{u}=\left\{v \in \bar{X}^{*} \mid\left(u u^{-1} v\right) \sim_{u}=u \sim_{u}\right\} .
$$

Let $v \in L_{u}=L(p, \Gamma, q)$ and $a, b \in \bar{X}^{*}$. If $a u u^{-1} v b \in L_{u}$ then, for some $r, s, t \in V(\Gamma)$,

$$
p \stackrel{a}{\longrightarrow} r \stackrel{u}{\longrightarrow} t \stackrel{u^{-1}}{\longrightarrow} r \stackrel{v}{\longrightarrow} s \stackrel{b}{\longrightarrow} q
$$

is a path in $\Gamma$ from $p$ to $q$. Since $u \in L(r, \Gamma, t)$ and $v \in L_{u}$, we also have that $v \in L(r, \Gamma, t)$ which, since $\Gamma$ is inverse, gives that $s=t$. It follows that $a u b \in L_{u}$. Conversely, suppose that $a u b \in L_{u}$. Then there is a path $p \stackrel{a}{\longrightarrow} r \stackrel{u}{\longrightarrow} s \stackrel{b}{\longrightarrow} q$ in $\Gamma$. Then $u \in L(r, \Gamma, s)$ so that $L(p, \Gamma, q)=L_{u} \subseteq L(r, \Gamma, s)$. Now, $v \in L(p, \Gamma, q)$ gives that $v \in L(r, \Gamma, s)$ so that $a u u^{-1} v b \in L(p, \Gamma, q)=L_{u}$. It follows that $\left(u u^{-1} v\right) \sim_{u}=u \sim_{u}$. Finally, if $\left(u u^{-1} v\right) \sim_{u}=u \sim_{u}$ then, as $u \in L_{u}$, we have that $\left(u u^{-1} v\right) \in L_{u}$ so that $v \in L_{u}$. It follows that $(p, \Gamma, q) \simeq \mathcal{S}\left(X, \sim_{u}, u\right)$ and so (i) $\Leftrightarrow$ (ii) is proved.

Suppose now that (ii) holds. If $L^{\prime} \in \Omega$ is such that $L_{u} \nsubseteq L^{\prime}$, then $L^{\prime} \notin \Omega(u)$ so that $u \notin L^{\prime}$. It follows that $u \notin \cup\left\{L^{\prime} \in \Omega \mid L \nsubseteq L^{\prime}\right\}$ and hence $L_{u} \nsubseteq \cup\left\{L^{\prime} \in \Omega \mid L_{u} \nsubseteq L^{\prime}\right\}$ giving (iii).

Assuming (iii), let $u \in L \backslash \cup\left\{L^{\prime} \in \Omega \mid L \nsubseteq L^{\prime}\right\}$. We have that $L \in \Omega(u)$ so that $\Omega(u) \neq \emptyset$. If $L^{\prime \prime} \in \Omega(u)$, then $u \in L^{\prime \prime}$ and so $L^{\prime \prime} \nsubseteq \cup\left\{L^{\prime} \in \Omega \mid L \nsubseteq L^{\prime}\right\}$. It follows that $L^{\prime \prime} \notin\left\{L^{\prime} \in \Omega \mid L \nsubseteq L^{\prime}\right\}$ so that $L \subseteq L^{\prime \prime}$ and $L=\cap \Omega(u)=L_{u} \in \Omega$, yielding (ii).

If $\Gamma$ is finite, the equivalence (i) $\Leftrightarrow$ (iii) provides an algorithm to decide whether or not $\Gamma$ arises as a Schützenberger graph, a result proved by Cowan and Reilly [1].

COROllary 5.2. [1] Given a finite inverse $\bar{X}$-graph $\Gamma$, it is decidable whether or not $\Gamma$ is a Schützenberger graph of some inverse monoid presentation.

Next we show that our choice of $\sim_{u}$ in the proof of (ii) $\Rightarrow$ (i) produces the smallest possible inverse monoid.

Proposition 5.3. Let $\Gamma$ be an inverse $\bar{X}$-graph such that there is $u \in \bar{X}^{*}$ with $L_{u} \in \Omega$, and let $\sim_{u}$ be the syntactic congruence of $L_{u}$ on $\bar{X}^{*}$. Then $\sim_{u}$ is the greatest inverse monoid congruence $\tau$ on $\bar{X}^{*}$ such that $\Gamma$ is a Schützenberger graph of the inverse monoid presentation $\operatorname{Inv}\langle X ; \tau\rangle$.

Proof. Let $\tau$ be a congruence on $\bar{X}^{*}$ such that $\Gamma$ is a Schützenberger graph of the inverse monoid presentation $\operatorname{Inv}\langle X ; \tau\rangle$. Since $L_{u} \in \Omega$, then $L_{u}=L(p, \Gamma, q)=$ $L(\mathcal{S}(X, \tau, w))$, for some $w \in \bar{X}^{*}$ and $p, q \in V(\Gamma)$. On the other hand $L(\mathcal{S}(X, \tau, w))=$ $\left\{v \in \bar{X}^{*} \mid v \tau \geq w \tau\right\}$, so that

$$
\begin{aligned}
v \tau v^{\prime} & \Leftrightarrow(a v b) \tau=\left(a v^{\prime} b\right) \tau \forall a, b \in \bar{X}^{*} \\
& \Rightarrow\left((a v b) \tau \geq w \tau \Leftrightarrow\left(a v^{\prime} b\right) \tau \geq w \tau\right) \forall a, b \in \bar{X}^{*} \\
& \Leftrightarrow\left((a v b) \in L_{u} \Leftrightarrow\left(a v^{\prime} b\right) \in L_{u}\right) \forall a, b \in \bar{X}^{*} \\
& \Leftrightarrow v \sim_{u} v^{\prime}
\end{aligned}
$$

so that $\tau \subseteq \sim_{u}$. 
Let $\Gamma$ be as in Proposition 5.3. By this result, in particular, it follows that, if $u, v \in \bar{X}^{*}$ are such that $L_{u}, L_{v} \in \Omega$, then $\sim_{u}=\sim_{v}$. If $\Gamma$ is a finite tree, then the set of congruences $\tau$ such that $\Gamma$ is a Schützenberger graph of the inverse monoid presentation $\operatorname{Inv}\langle X ; \tau\rangle$, is the interval $\left[\rho, \sim_{u}\right]$. If $\Gamma$ is not a tree, then there is no smallest congruence $\tau$ as above, as will follow from the next result.

Proposition 5.4. Let $\Gamma$ be an inverse $\bar{X}$-graph such that $\left(S^{\prime}\right)$ holds. If $\Upsilon$ is the set of inverse monoid congruences $\tau$ on $\bar{X}^{*}$ such that $\Gamma$ is a Schützenberger graph of $\operatorname{Inv}\langle X ; \tau\rangle$, then

$$
\rho=\cap \Upsilon .
$$

Proof. The inclusion from right to left is trivial. To prove that $\cap \Upsilon \subseteq \rho$, we may assume that $\Gamma$ is not a tree. Let $a, b \in \bar{X}^{*}$ be such that $(a, b) \notin \rho$. Let $\Gamma=\mathcal{S} \Gamma(X, \tau, u)$ for some inverse monoid congruence $\tau$ on $\bar{X}^{*}$ and $u \in \bar{X}^{*}$. For every $k>0$, we define

$$
J_{k}=\left\{v \in \bar{X}^{*}|| \mathrm{MT}(v) \mid \geq k\right\}
$$

and $\tau_{k}=\rho \cup\left(\tau \cap\left(J_{k} \times J_{k}\right)\right)$. It is a routine exercise to check that $\tau_{k}$ is an inverse monoid congruence on $\bar{X}^{*}$. If we choose $n>\max \{|\operatorname{MT}(a)|,|\operatorname{MT}(b)|\}$, then clearly $(a, b) \notin \tau_{n}$. Hence to show that $\cap \Upsilon \subseteq \rho$ it is enough to prove that $\Gamma$ is a Schützenberger graph for $\operatorname{Inv}\left\langle X ; \tau_{n}\right\rangle$.

Since $\Gamma$ is not a tree, there exists $r \in V(\Gamma)$ and $w \in L(r, \Gamma, r)$ such that $w \iota \neq 1$. Let $p, q \in V(\Gamma)$ be such that $\mathcal{S}(X, \tau, u)=(p, \Gamma, q)$. Then there is a path $q \stackrel{v}{\longrightarrow} r$ in $\Gamma$. For every $i \geq 0$, we have $u v w^{i} v^{-1} \in L(p, \Gamma, q)=L(\mathcal{S}(X, \tau, u))$ so that Proposition 2.3 yields

$$
u \tau=\left(u u^{-1} u v w^{i} v^{-1}\right) \tau=\left(u v w^{i} v^{-1}\right) \tau
$$

and so $\mathcal{S}(X, \tau, u)=\mathcal{S}\left(X, \tau, u v w^{i} v^{-1}\right)$. Since $w \iota \neq 1$, there is $m \geq 0$ such that $u v w^{m} v^{-1} \in J_{n}$. Let $z=u v w^{m} v^{-1}$. We will see that $\mathcal{S}(X, \tau, z)=\mathcal{S}\left(X, \tau_{n}, z\right)$, which yields that $\Gamma$ is a Schützenberger graph for $\operatorname{Inv}\left\langle X ; \tau_{n}\right\rangle$. To do so, we show that $L(\mathcal{S}(X, \tau, z))=L\left(\mathcal{S}\left(X, \tau_{n}, z\right)\right)$. The converse inclusion is immediate. Assume now that $c \in L(\mathcal{S}(X, \tau, z))$. Then $z \tau\left(z z^{-1} c\right)$ and, since $z \in J_{n}$, we have $z z^{-1} c \in J_{n}$. Thus $z \tau_{n}\left(z z^{-1} c\right)$ and so $c \in L\left(\mathcal{S}\left(X, \tau_{n}, z\right)\right)$. Hence $L(\mathcal{S}(X, \tau, z))=L\left(\mathcal{S}\left(X, \tau_{n}, z\right)\right)$, as required.

6. Schützenberger graphs of E-unitary and Clifford monoids. We consider in this section the problem of characterizing a Schützenberger graph of particular types of monoids: Clifford monoids and E-unitary monoids. In the case of Clifford monoids, the provided conditions also show that, given an inverse graph, it is decidable whether or not that graph is a Schützenberger graph of a Clifford monoid. This is not the case with E-unitary monoids and, since this is far more complicated and it involves completely different arguments, it is treated separately in the next section.

Let $\Gamma$ be an inverse $\bar{X}$-graph satisfying $\left(S^{\prime}\right)$. From Theorem 5.1, we have that $\Gamma$ is a Schützenberger graph of the inverse monoid presentation $\operatorname{Inv}\left\langle X ; \sim_{u}\right\rangle$.

Lemma 6.1 For every $v, w \in \bar{X}^{*}$, we have

$$
v \sim_{u} w \text { iff }(v \in L(r, \Gamma, s) \Leftrightarrow w \in L(r, \Gamma, s)) \forall r, s \in V(\Gamma) .
$$


Proof. Let $L_{u}=L(p, \Gamma, q)$, with $p, q \in V(\Gamma)$. Suppose that $v \sim_{u} w$. If $v \in L(r, \Gamma, s)$ then, since $\Gamma$ is connected, there are $a, b \in \bar{X}^{*}$ such that $a \in L(p, \Gamma, r)$ and $b \in L(s, \Gamma, q)$. Thus $a v b \in L(p, \Gamma, q)$ that is, $a v b \in L_{u}$. It follows that $a w b \in L_{u}$ which, since $\Gamma$ is inverse, gives that $w \in L(r, \Gamma, s)$. By symmetry, the second condition follows.

Suppose now that the second condition holds. If $a v b \in L_{u}$ then $a v b \in L(p, \Gamma, q)$ and thus $p \stackrel{a}{\longrightarrow} r \stackrel{v}{\longrightarrow} s \stackrel{b}{\longrightarrow} q$ is a path in $\Gamma$, for some $r, s \in V(\Gamma)$. By assumption, $v \in L(r, \Gamma, s)$ yields that $w \in L(r, \Gamma, s)$ giving that $p \stackrel{a w b}{\longrightarrow} q$ is a path in $\Gamma$ and so $a w b \in L(p, \Gamma, q)=L_{u}$. Again, by symmetry, the implication follows.

THEOREM 6.2. If $\left(S^{\prime}\right)$ holds, the following conditions are equivalent:

(i) $\Gamma$ is a Schützenberger graph of some Clifford monoid;

(ii) for all $L, L^{\prime} \in \Omega$, $\operatorname{Pref}(L)=\operatorname{Pref}\left(L^{\prime}\right)$;

(iii) for all $p, q \in V(\Gamma), L(p, \Gamma, p)=L(q, \Gamma, q)$.

Proof. Let $M=\bar{X}^{*} / \sim_{u}$. Clearly Theorem 4.2 gives that (i) implies (ii) and (iii). Now, using Lemma 6.1 , it is easily seen that essentially the same proof as that of Theorem 4.2 yields that (ii) $\Rightarrow$ (iii) and (iii) $\Rightarrow$ (i).

Corollary 6.3. Given a finite inverse $\bar{X}$-graph $\Gamma$, it is decidable whether or not $\Gamma$ is a Schützenberger graph of a Clifford monoid.

Proof. The languages $L(p, \Gamma, p)$, with $p \in V(\Gamma)$, constitute a finite family of rational languages and we can decide whether any two of them are equal.

THEOREM 6.4. If $\left(S^{\prime}\right)$ holds, the following conditions are equivalent:

(i) $\Gamma$ is a Schützenberger graph of some E-unitary monoid;

(ii) $\Gamma$ embeds in the Cayley graph of some group.

Proof. (i) $\Rightarrow$ (ii) follows from Theorem 4.1. Now, suppose that $\Gamma$ embeds in the Cayley graph $\Gamma^{\prime}$ of a group. Let $L_{u}=L(p, \Gamma, q)$, with $p, q \in V(\Gamma)$. Define $\tau$ to be the congruence on $\bar{X}^{*}$ generated by $\rho$ and the relations $u=u a a^{-1}$ and $b=b b^{-1}$, where $a \in L(q, \Gamma, r)$, for some $r \in V(\Gamma)$, and $b \in \bar{X}^{*}$ labels a loop in $\Gamma^{\prime}$. Next we show that $\tau \subseteq \sim_{u}$. In view of Lemma 6.1, we only have to prove that

$$
(v \in L(t, \Gamma, s) \Leftrightarrow w \in L(t, \Gamma, s)) \forall t, s \in V(\Gamma)
$$

whenever $v=w$ is a generator of $\tau$. This is trivial when $(v, w) \in \rho$ and, since $\Gamma$ embeds in $\Gamma^{\prime}$, also for relations of the form $b=b b^{-1}$, where $b \in \bar{X}^{*}$ labels a loop in $\Gamma^{\prime}$. Assume now that $v=u$ and $w=u a a^{-1}$, where $a \in L(q, \Gamma, r)$, for some $r \in V(\Gamma)$. Let $t, s \in V(\Gamma)$. The inclusion from left to right is trivial. Suppose now that $u \in L(t, \Gamma, s)$. Then $L(p, \Gamma, q)=L_{u} \subseteq L(t, \Gamma, s)$ and so $u a a^{-1} \in L(p, \Gamma, q)$ yields $u_{a a}{ }^{-1} \in L(t, \Gamma, s)$, as required. Thus $\tau \subseteq \sim_{u}$.

We first show that $(p, \Gamma, q) \simeq \mathcal{S}(X, \tau, u)$, by seeing that $L(p, \Gamma, q)=L(\mathcal{S}(X, \tau, u))$. We have that $L(p, \Gamma, q)=L_{u}=\left\{v \in \bar{X}^{*} \mid u \sim_{u}\left(u u^{-1} v\right)\right\}$ and $L(\mathcal{S}(X, \tau, u))=$ $\left\{v \in \bar{X}^{*} \mid u \tau\left(u u^{-1} v\right)\right\}$. Since $\tau \subseteq \sim_{u}$ we have that $L(\mathcal{S}(X, \tau, u)) \subseteq L(p, \Gamma, q)$. Now, let $v \in L(p, \Gamma, q)$. Then $a=u^{-1} v \in L(q, \Gamma, q)$ so that $a$ labels a loop in $\Gamma^{\prime}$. Hence $u \tau=\left(u a a^{-1}\right) \tau$ and $a \tau=\left(a a^{-1}\right) \tau$ which gives that $u \tau=(u a) \tau=\left(u u^{-1} v\right) \tau$ and $v \in L(\mathcal{S}(X, \tau, u))$. Hence $L(p, \Gamma, q)=L(\mathcal{S}(X, \tau, u))$. 
To prove that $\tau$ is E-unitary, we show that, for all $v \in \bar{X}^{*}, \mathcal{S} \Gamma(X, \tau, v)$ embeds in the Cayley graph $\mathcal{S} \Gamma(X, \sigma, v)$, where $\sigma$ denotes the least group congruence on $\bar{X}^{*}$ containing $\tau$. If $r \tau \in V(\mathcal{S} \Gamma(X, \tau, v))$, then there is $a \in \bar{X}^{*}$ such that $a \in L(v \tau$, $\mathcal{S} \Gamma(X, \tau, v), r \tau)$. If $b \in \bar{X}^{*}$ is such that $b \in L(v \tau, \mathcal{S} \Gamma(X, \tau, v), r \tau)$, then $r \tau=(v a) \tau=(v b) \tau$ and thus $r \sigma=(v a) \sigma=(v b) \sigma$, for $\tau \subseteq \sigma$. Since $\sigma$ is a group congruence, it follows that $a \sigma=b \sigma$. Let $(r \tau) \varphi=a \sigma$. We have thus defined a map $\varphi: V(\mathcal{S} \Gamma(X, \tau, v)) \rightarrow$ $V(\mathcal{S} \Gamma(X, \sigma, v))$, which is clearly a graph homomorphism.

Now, if $(r \tau) \varphi=(s \tau) \varphi$, then $v \tau \stackrel{a}{\longrightarrow} r \tau$ and $v \tau \stackrel{b}{\longrightarrow} s \tau$ are paths in $\mathcal{S} \Gamma(X, \tau, v)$ and $a b^{-1}$ labels a loop in $\mathcal{S} \Gamma(X, \sigma, v)$. It follows that $\left(a b^{-1}\right) \tau=\left(a b^{-1} b a^{-1}\right) \tau$ and so $r \tau=s \tau$. Hence $\varphi$ is injective and $\mathcal{S}(X, \tau, v)$ embeds in the Cayley graph $\mathcal{S} \Gamma(X, \sigma, v)$. From Theorem 4.1, it follows that $\tau$ is E-unitary, and (ii) $\Rightarrow$ (i).

7. Groups and E-unitary inverse monoids. Let $\pi$ be the congruence on $\bar{X}^{*}$ induced by $\iota$. Then $F G(X)=\bar{X}^{*} / \pi$ is the free group on $X$.

A word $w \in \bar{X}^{*}$ is said to be cyclically reduced if $w^{2} \in \bar{X}^{*} \iota$. Given $w \in \bar{X}^{*}$, there exist unique $u, v \in \bar{X}^{*} \iota$ such that $w \iota=u v u^{-1}$ and $v$ is cyclically reduced. We denote $v$ by $w \gamma$.

A group presentation is a formal expression of the form $\mathcal{P}=\mathrm{Gp}\langle X ; R\rangle$, where $X$ is a set and $R \subseteq \bar{X}^{*}$. The group defined by this presentation is the quotient $F G(X) /\langle R \pi\rangle_{X}$, where $\langle R \pi\rangle_{X}$ denotes the normal closure of $R \pi$ in $F G(X)$.

Given a finite group presentation $\mathcal{P}=\mathrm{Gp}\langle X ; R\rangle$, we denote by $\mathcal{C}(X, R)$ the Cayley graph of $\mathcal{P}$. We define also

$$
c(R)=\left\{x \in X: x \text { or } x^{-1} \text { occurs in some } r \in R\right\} .
$$

THEOREM 7.1. It is undecidable whether or not an arbitrary finite inverse graph embeds in the Cayley graph of some group.

Proof. Suppose that this problem is decidable. We will show that, given this, we can decide whether or not an arbitrary finite group presentation defines a trivial group, contradicting a well-known undecidability result [4].

Let $\mathcal{P}=\mathrm{Gp}\langle X ; R\rangle$ be a finite group presentation and let $G=F G(X) /\langle R \pi\rangle_{X}$ denote the group defined by $\mathcal{P}$. Let $R=\left\{r_{1}, \ldots, r_{m}\right\}$. Without loss of generality, we can make the following assumptions:

$\left(A_{1}\right) \quad m \geq 1$;

$\left(A_{2}\right) \quad r_{i} \neq 1$ for every $i \in\{1, \ldots, m\}$;

$\left(A_{3}\right) \quad r_{i}$ is cyclically reduced for every $i \in\{1, \ldots, m\}$;

$\left(A_{4}\right) \quad c(R)=X$.

We can assume that $\left(A_{4}\right)$ holds, otherwise $G$ would have a nontrivial free group as a free factor [4], and would therefore be nontrivial.

Let $z$ denote a letter not belonging to $\bar{X}$, and write $X^{\prime}=X \cup\{z\}$ and $\overline{X^{\prime}}=X^{\prime} \cup X^{\prime-1}$. Let $\Gamma=\Gamma(R)$ denote the inverse $\overline{X^{\prime}}$-graph described by
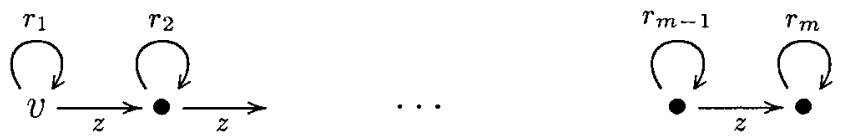

Let $L$ denote the set of all labels of loops from $v$ to $v$ in $\Gamma$. We show that 


$$
L \pi \subseteq\langle R \pi\rangle_{X^{\prime}}
$$

Let $u \in L$. By a well-known result [4], $u \pi$ belongs to the subgroup $S$ of $F G\left(X^{\prime}\right)$ generated by

$$
\left\{r_{1} \pi,\left(z r_{2} z^{-1}\right) \pi, \ldots,\left(z^{m-1} r_{m} z^{-m+1}\right) \pi\right\}
$$

hence $u \pi \in\langle R \pi\rangle_{X^{\prime}}$ and (3) holds.

We define $\varphi: \Gamma \rightarrow \mathcal{C}\left(X^{\prime}, R\right)$ as follows. Let $p \in V(\Gamma)$. We choose a path $v \stackrel{a}{\longrightarrow} p$ in $\Gamma$. Since $\mathcal{C}\left(X^{\prime}, R\right)$ is complete, there exists a path of the form $1 \stackrel{a}{\longrightarrow} p^{\prime}$ in $\mathcal{C}\left(X^{\prime}, R\right)$. We take $p \varphi=p^{\prime}$. To show that $\varphi$ is well-defined, suppose that $v \stackrel{b}{\longrightarrow} p$ is another path in $\Gamma$ and $1 \stackrel{b}{\longrightarrow} p^{\prime \prime}$ a path in $\mathcal{C}\left(X^{\prime}, R\right)$. Then $a b^{-1}$ labels a loop in $\Gamma$, hence $a b^{-1} \in L$. By (3), it follows that $\left(a b^{-1}\right) \pi \in\langle R \pi\rangle_{X^{\prime}}$ and so $a b^{-1}$ labels a loop at every vertex in $\mathcal{C}\left(X^{\prime}, R\right)$. In particular, we obtain $p^{\prime}=p^{\prime \prime}$. Let $(p, y, q) \in E(\Gamma)$. Since $\mathcal{C}\left(X^{\prime}, R\right)$ is complete, we have an edge of the form $\left(p \varphi, y, q^{\prime}\right)$ in $\mathcal{C}\left(X^{\prime}, R\right)$. It follows from the definition that $q^{\prime}=q \varphi$, hence $\varphi$ is indeed a graph homomorphism. We show that

$\Gamma$ embeds in the Cayley graph of some group $\Leftrightarrow \varphi$ is injective.

The inclusion from left to right is of course trivial. Let us assume that there exists an embedding $\psi: \Gamma \rightarrow \mathcal{C}(Y, S)$ for some group presentation $\operatorname{Gp}\langle Y ; S\rangle$. Since every Cayley graph has a transitive automorphism group, we may assume that $v \psi=1$. Since we are assuming that all letters of $X^{\prime}$ label edges in $\Gamma$, we have

$$
\overline{X^{\prime}} \subseteq Y \cup Y^{-1}
$$

Let $i \in\{1, \ldots, m\}$. Since $\Gamma$ embeds in $\mathcal{C}(Y, S)$, it follows that $r_{i}$ labels a loop in $\mathcal{C}(Y, S)$ and, by definition of Cayley graph, this yields $r_{i} \pi \in\langle S \pi\rangle_{Y}$. Thus

$$
R \pi \subseteq\langle S \pi\rangle_{Y}
$$

To show that $\varphi$ is injective, we assume that $p \varphi=q \varphi$ for some $p, q \in V(\Gamma)$. Let $\nu \stackrel{a}{\longrightarrow} p$ and $v \stackrel{b}{\longrightarrow} q$ be paths in $\Gamma$. Then we have a path of the form

$$
p \psi \stackrel{a^{-1} b}{\longrightarrow} q \psi
$$

in $\mathcal{C}(Y, S)$. Since $p \varphi=q \varphi, a^{-1} b$ labels a loop in $\mathcal{C}\left(X^{\prime}, R\right)$. Thus $a^{-1} b \in\langle R \pi\rangle_{X^{\prime}}$. By (5) and (6), we obtain $\langle R \pi\rangle_{X^{\prime}} \subseteq\langle S \pi\rangle_{Y}$. Hence $\left(a^{-1} b\right) \pi \in\langle S \pi\rangle_{Y}$ and so $a^{-1} b$ labels a loop at every vertex in $\mathcal{C}(Y, S)$. Since (7) is a path in $\mathcal{C}(Y, S)$ and $\mathcal{C}(Y, S)$ is deterministic, we obtain $p \psi=q \psi$. By hypothesis, $\psi$ is injective, hence $p=q$ and so $\varphi$ is also an embedding. Therefore (4) holds.

Let $\Lambda=\Lambda(R)$ denote the set of all nonempty proper factors of words of $R$. Next we show that

$$
\varphi \text { is injective } \Leftrightarrow(\Lambda \pi) \cap\langle R \pi\rangle_{X^{\prime}}=\emptyset \text {. }
$$

Assume that $\varphi$ is injective and let $u \in \Lambda$. Then $u$ is a nonempty proper factor of some $r_{i}$ and so $u$ labels a path in $\Gamma$ which is not a loop. Suppose that $u \pi \in\langle R \pi\rangle_{X^{\prime}}$. Then $u$ labels a loop at every vertex in $\mathcal{C}\left(X^{\prime}, R\right)$. Since $\varphi$ is injective, every path labelled by $u$ in $\Gamma$ must be a loop, a contradiction. Thus $u \pi \notin\langle R \pi\rangle_{X^{\prime}}$ and $(\Lambda \pi) \cap\langle R \pi\rangle_{X^{\prime}}=\emptyset$. 
Conversely, assume that $\varphi$ is not injective. Then there exist $p, q \in V(\Gamma)$ such that $p \varphi=q \varphi$ and $p \neq q$. We can split $V(\Gamma)$ into $m$ disjoint classes according to the $m$ main loops of $\Gamma$. Suppose that $p$ and $q$ lie in different classes. Without loss of generality, we may assume that there exists a path in $\Gamma$ of the form

$$
p \stackrel{a z^{k} b}{\longrightarrow} q
$$

with $k>0$ and $a, b \in \bar{X}^{*}$. Let $\stackrel{\stackrel{c}{\longrightarrow}}{\longrightarrow} p$ be a path in $\Gamma$. Then $1 \stackrel{c}{\longrightarrow} p \varphi$ and

$$
1 \stackrel{c a z^{k} b}{\longrightarrow} q \varphi
$$

are paths in $\mathcal{C}\left(X^{\prime}, R\right)$ and so $a z^{k} b$ labels a loop at every vertex in $\mathcal{C}\left(X^{\prime}, R\right)$. Hence $\left(a z^{k} b\right) \pi \in\langle R \pi\rangle_{X^{\prime}}$. This is impossible, since $R \subseteq \bar{X}^{*}$ implies that, whenever $u \pi \in\langle R \pi\rangle_{X^{\prime}}$, the number of occurrences of $z$ in $u$ must match the number of occurrences of $z^{-1}$. Thus $p$ and $q$ must lie in the same main loop of $\Gamma$, say, the one labelled by $r_{i}$. Since $p \neq q$, we may write $r_{i}=$ sut, where $u \in L(p, \Gamma, q)-\left\{1, r_{i}\right\}$. Clearly, $u \in \Lambda$. Since

$$
v \stackrel{z^{i-1} s}{\longrightarrow} p
$$

is a path in $\Gamma$, then

$$
1 \stackrel{z^{i-1} s}{\longrightarrow} p \varphi \text { and } 1 \stackrel{z^{i-1} s u}{\longrightarrow} q \varphi
$$

are paths in $\mathcal{C}\left(X^{\prime}, R\right)$ and so $u$ labels a loop at every vertex in $\mathcal{C}\left(X^{\prime}, R\right)$. Hence $u \pi \in(\Lambda \pi) \cap\langle R \pi\rangle_{X^{\prime}}$. Thus $(\Lambda \pi) \cap\langle R \pi\rangle_{X^{\prime}} \neq \emptyset$ and (8) holds.

Next we define $\mathcal{F}$ as the smallest family of group presentations satisfying the following conditions.

- $\mathcal{P}=\mathrm{Gp}\langle X ; R\rangle \in \mathcal{F}$.

- If $\operatorname{Gp}\langle X ; S\rangle \in \mathcal{F}, \quad s=s_{1} s_{2} s_{3} \in S$ and $s_{2} \notin\{1, s\}$, then $\operatorname{Gp}\langle X ;(S-\{s\}) \cup$ $\left.\left\{\left(s_{1} s_{3}\right) \gamma, s_{2} \gamma\right\}\right\rangle \in \mathcal{F}$.

Note that, for every $\operatorname{Gp}\langle X ; S\rangle \in \mathcal{F}, S$ is finite. Moreover, since we are assuming that $R$ is reduced, $S$ is certainly reduced. The inequality $\left|\left(s_{1} s_{3}\right) \gamma\right|+\left|s_{2} \gamma\right| \leq|s|$ yields

$$
\sum_{s \in S}|s| \leq \sum_{i=1}^{m}\left|r_{i}\right|
$$

for every $\operatorname{Gp}\langle X ; S\rangle \in \mathcal{F}$. Thus $\mathcal{F}$ is finite and it is immediate that it is effectively constructible.

Next we show that conditions $\left(A_{1}\right)-\left(A_{3}\right)$ are valid for every $\operatorname{Gp}\langle X ; S\rangle \in \mathcal{F}$. Conditions $\left(A_{1}\right)$ and $\left(A_{3}\right)$ are trivially inherited in $\mathcal{F}$, and the requirement $s_{2} \notin\{1, s\}$ guarantees that $\left(A_{2}\right)$ is inherited as well.

We show that the group $G$ defined by $\mathcal{P}$ is trivial if and only if, for every $\mathrm{Gp}\langle X ; S\rangle \in \mathcal{F},\left(A_{4}\right)$ and the following condition holds.

$\left(A_{5}\right) \quad \Gamma(S)$ does not embed in the Cayley graph of some group or, for every

$$
x \in X, x \in S \text { or } x^{-1} \in S \text {. }
$$

We start by proving that

$$
\langle S \pi\rangle_{X} \subseteq\left\langle\left((S-\{s\}) \cup\left\{\left(s_{1} S_{3}\right) \gamma, s_{2} \gamma\right\}\right) \pi\right\rangle_{X}
$$


whenever $\operatorname{Gp}\langle X ; S\rangle \in \mathcal{F}, s=s_{1} s_{2} s_{3} \in S$ and $s_{2} \notin\{1, s\}$. Writing $\left(s_{1} s_{3}\right) \iota=a\left(\left(s_{1} s_{3}\right) \gamma\right) a^{-1}$ and $s_{2}=b\left(s_{2} \gamma\right) b^{-1}$ for the appropriate $a, b \in \bar{X}^{*}$, we have

$$
\begin{aligned}
s \pi & =\left(s_{1} s_{2} s_{3}\right) \pi=\left(s_{1} s_{2} s_{1}^{-1}\right) \pi\left(s_{1} s_{3}\right) \pi=\left(s_{1} s_{2} s_{1}^{-1}\right) \pi\left(\left(s_{1} s_{3}\right) \iota\right) \pi \\
& =\left(s_{1} b\left(s_{2} \gamma\right) b^{-1} s_{1}^{-1}\right) \pi\left(a\left(\left(s_{1} s_{3}\right) \gamma\right) a^{-1}\right) \pi,
\end{aligned}
$$

and it becomes clear that $s \pi \in\left\langle\left((S-\{s\}) \cup\left\{\left(s_{1} s_{3}\right) \gamma, s_{2} \gamma\right\}\right) \pi\right\rangle_{X}$. Thus (9) holds.

Assume now that $G$ is trivial. By (9), it follows easily that every $\operatorname{Gp}\langle X ; S\rangle \in \mathcal{F}$ defines a trivial group. We have already remarked that conditions $\left(A_{1}\right)-\left(A_{3}\right)$ are valid for every $\operatorname{Gp}\langle X ; S\rangle \in \mathcal{F}$. With respect to $\left(A_{4}\right)$, it is enough to observe that $c(S)=X$ follows from $\mathrm{Gp}\langle X ; S\rangle$ defining the trivial group, otherwise the group defined by $\operatorname{Gp}\langle X ; S\rangle$ would have a nontrivial free group as a free factor and would therefore be nontrivial, a contradiction.

Let $\mathrm{Gp}\langle X ; S\rangle \in \mathcal{F}$. Suppose that there exists $x \in X$ such that $x, x^{-1} \notin S$. Since conditions $\left(A_{1}\right)-\left(A_{4}\right)$ hold, we can apply (4) and (8) to $\Gamma(S)$. Hence we want to prove that $((\Lambda(S)) \pi) \cap\langle S \pi\rangle_{X^{\prime}} \neq \emptyset$. Since $x \in X=c(S)$, we have $a x^{\varepsilon} b \in S$ for some $\varepsilon \in\{-1,1\}$ and $a, b \in \bar{X}^{*} \iota$. Since $x, x^{-1} \notin S$, we have $a b \neq 1$, and so $x^{\varepsilon} \in \Lambda(S)$. Since $\operatorname{Gp}\langle X ; S\rangle \in \mathcal{F}$ defines a trivial group, we have $\bar{X}^{*} \pi \subseteq\langle S \pi\rangle_{X^{\prime}}$. Thus $x^{\varepsilon} \pi \in((\Lambda(S)) \pi) \cap\langle S \pi\rangle_{X^{\prime}}$ and so $\left(A_{5}\right)$ is satisfied.

Conversely, assume that $\left(A_{4}\right)$ and $\left(A_{5}\right)$ are satisfied for every $\operatorname{Gp}\langle X ; S\rangle \in \mathcal{F}$. We define a sequence $\mathrm{Gp}\left\langle X ; S_{0}\right\rangle, \mathrm{Gp}\left\langle X ; S_{1}\right\rangle, \ldots$ in $\mathcal{F}$ with $\left\langle S_{j} \pi\right\rangle_{X}=\langle R \pi\rangle_{X}$ for every $j \geq 0$ as follows. Let $S_{0}=R$. Assume that $S_{j}$ is defined. If $x \in S_{j}$ or $x^{-1} \in S_{j}$ for every $x \in X$, it is clear that $\langle R \pi\rangle_{X}=\left\langle S_{j} \pi\right\rangle_{X}=\bar{X}^{*} \pi$ and so $G$ is trivial as required. Suppose that there exists $x \in X$ such that $x, x^{-1} \notin S_{j}$. By $\left(A_{5}\right), \Gamma\left(S_{j}\right)$ does not embed in the Cayley graph of some group. Since conditions $\left(A_{1}\right)-\left(A_{4}\right)$ are valid for $\operatorname{Gp}\left\langle X ; S_{j}\right\rangle \in \mathcal{F}$, we can apply (4) and (8) to $\Gamma\left(S_{j}\right)$ and conclude that $\left(\left(\Lambda\left(S_{j}\right)\right) \pi\right) \cap\left\langle S_{j} \pi\right\rangle_{X^{\prime}} \neq \emptyset$. Thus there exist $a, u, b \in \bar{X}^{*} \iota$ such that

$$
a u b \in S_{j}, \quad(a b) \iota \neq 1, \quad u \neq 1, \quad u \pi \in\left\langle S_{j} \pi\right\rangle_{X^{\prime}} .
$$

We define

$$
S_{j+1}=\left(S_{j}-\{a u b\}\right) \cup\{(a b) \gamma, u \gamma\}
$$

By definition of $\mathcal{F}$, we have $\operatorname{Gp}\left\langle X ; S_{j+1}\right\rangle \in \mathcal{F}$. By (9), we obtain $\left\langle S_{j} \pi\right\rangle_{X} \subseteq\left\langle S_{j+1} \pi\right\rangle_{X}$. Since $S_{j} \cup\{u\} \subseteq \bar{X}^{*}$ and $u \pi \in\left\langle S_{j} \pi\right\rangle_{X^{\prime}}$, it follows easily that $u \pi \in\left\langle S_{j} \pi\right\rangle_{X}$ and so $u \gamma \pi \in\left\langle S_{j} \pi\right\rangle_{X}$. We also obtain

$$
(a b) \pi=\left(\left(a u a^{-1}\right)^{-1}(a u b)\right) \pi \in\left\langle S_{j} \pi\right\rangle_{X},
$$

hence $(a b) \gamma \pi \in\left\langle S_{j} \pi\right\rangle_{X}$ and $\left\langle S_{j+1} \pi\right\rangle_{X}=\left\langle S_{j} \pi\right\rangle_{X}=\langle R \pi\rangle_{X}$.

We have thus built a sequence $\operatorname{Gp}\left\langle X ; S_{0}\right\rangle, \mathrm{Gp}\left\langle X ; S_{1}\right\rangle, \ldots$ in $\mathcal{F}$ with $\left\langle S_{j} \pi\right\rangle_{X}=\langle R \pi\rangle_{X}$ for every $j \geq 0$. It follows from the definition of the sequence that, for every $j \geq 0$, we have either

$$
\sum_{s \in S_{j+1}}|s|<\sum_{s^{\prime} \in S_{j}}\left|s^{\prime}\right|
$$

or

$$
\sum_{s \in S_{j+1}}|s|=\sum_{s^{\prime} \in S_{j}}\left|s^{\prime}\right| \text { and }\left|S_{j+1}\right|>\left|S_{j}\right| .
$$


Since $1 \notin S_{j}$ for every $j$, it follows easily that the sequence must be finite. Thus there exists $k \geq 0$ such that $x \in S_{k}$ or $x^{-1} \in S_{k}$ for every $x \in X$. Since $\langle R \pi\rangle_{X}=\left\langle S_{k} \pi\right\rangle_{X}=\bar{X}^{*} \pi$, it follows that $G$ is trivial.

We have just proved that $G$ is trivial if and only if, for every $\operatorname{Gp}\langle X ; S\rangle \in \mathcal{F}$, conditions $\left(A_{4}\right)$ and $\left(A_{5}\right)$ hold. We have already remarked that $\mathcal{F}$ is a finite effectively constructible set, and $\left(A_{4}\right)$ is certainly decidable. If we could decide whether or not an arbitrary finite inverse graph embeds in the Cayley graph of some group, it would follow that we could decide whether or not an arbitrary finite group presentation defines a trivial group, a contradiction. Therefore our problem is undecidable.

COROLlARY 7.2. It is undecidable whether or not an arbitrary finite inverse graph is a Schützenberger graph of some E-unitary inverse monoid.

Proof. Assume that this problem is decidable. If we consider graphs of the form $\Gamma=\Gamma(R)$ as in the proof of Theorem 7.1, we have that $\Gamma$ is a Schützenberger graph for some inverse monoid presentation, since $L_{z^{m}} \in \Omega$. By Theorem 6.4, we would be able to decide whether or not $\Gamma$ embeds in the Cayley graph of a group, contradicting Theorem 7.1. Thus the considered problem is undecidable.

\section{REFERENCES}

1. D. Cowan and N. R. Reilly, Characterizations of Schützenberger graphs in terms of their automorphism groups and fundamental groups, Glasgow Math. J. 35 (1993), 275-291.

2. S. Eilenberg, Automata, languages and machines (Vol. A) (Academic Press, New York, 1974).

3. J. M. Howie, An introduction to semigroup theory (Academic Press, London, 1976). 1977).

4. R. C. Lyndon and P. E. Schupp, Combinatorial group theory (Springer-Verlag, Berlin,

5. S. W. Margolis and J. C. Meakin, Inverse monoids, trees and context-free languages, Trans. Amer. Math. Soc. 335 (1993), 259-276. 404.

6. W. D. Munn, Free inverse semigroups, Proc. London Math. Soc. (3) 29 (1974), 385-

7. M. Petrich, Inverse semigroups (Wiley, New York, 1984).

8. R. L. Ruyle, Pseudovarieties of inverse monoids, Ph.D. thesis, University of Nebraska, 1997.

9. J. B. Stephen, Presentations of inverse monoids, J. Pure Appl. Alg. 63 (1990), 81-112. 